\title{
Aggregation \\ in Models with Quantity Constraints: The CES Aggregation Function
}

\author{
Horst Entorf \\ and \\ Henri R. Sneessens ${ }^{1}$
}

December 1998

1 The first author is at the Institut für Volkswirtschaftslehre, Universität Würzburg; the second is at IRES, Université Catholique de Louvain, and Faculté Libre des Sciences Economiques, Lille. The second author has benefited from the financial support of the Belgian Program on Interuniversity Poles of Attraction. We are grateful to Inge Mattern for skillful assistance and to anonymous referees for most helpful comments and suggestions on an earlier draft. The usual disclaimer of course applies. 


\begin{abstract}
This paper is devoted to the problem of aggregation in models with quantity constraints. The focus is on quantity rationing macroeconomic (QRM) models where the micromarket outcome can be written as the minimum of several variables and where the diversity of situations across micromarkets is explicitly recognized. The aggregation result given in this paper generalizes that of Lambert (1988) to employment functions with more than two components, and leads to approximate aggregate functions of the CES variety. The approximation used can accomodate general variance-covariance structures. Simulation experiments show that the approximation error remains within reasonable bounds $(1-4 \%)$. It thus seems that the CES formulation can accomodate a large variety of situations. It remains in particular valid when the (restrictive) conditions required to obtain the CES function as an exact result (independently and identically distributed Weibull variables) are not satisfied.
\end{abstract}




\section{Introduction}

This paper is devoted to the problem of aggregation in models with quantity constraints and heterogeneous agents. Quantity constraints may play an important role in macroeconomic analysis, especially as determinants of unemployment, as illustrated in Barro-Grossman (1971) and Malinvaud (1977). These early "quantity rationing macroeconomic" (QRM) models have since then been extended to have representations of the wage and price formation processes based on sound microfoundations similar to those used in "New Keynesian Macroeconomics" (monopolistic competition and wage bargaining; see for instance Sneessens-Drèze, 1986). Quantity constraints may also play a crucial role in other setups, including credit rationing on imperfect capital markets, stockouts and inventory behaviours, demand uncertainty and labour and/or capital underutilization, especially in imperfect competition setups with real and/or nominal rigidities. The existence of such quantity constraints may affect significantly the dynamics of the aggregate economy (the propagation mechanism) in response to external shocks. See for instance Bernanke-Gertler (1989), and Carlstrom-Fuerst (1997), for credit constraints; Kahn (1987) and BlanchardFischer (1989, ch.6) for stockouts; Fagnart et al. (1997) for capacity utilization.

The focus in this paper is on aggregation over heterogeneous agents when the individual agent's outcome can be written as the minimum of several variables (for instance, employment as the minimum of demand and supply). Because different agents may face different constraints, the minimum rule no longer applies at the aggregate level (as in the early disequilibrium literature). For example on the labour market, because some micromarkets may be constrained by demand while others are constrained by supply, excess demand (vacancies) and excess supply (unemployment) situations will coexist at the aggregate level. This feature is most interesting, as it allows one to introduce explicitly and rigorously into the analysis features like the proportion of sales-, capacity-, or labour-constrained firms, as they appear in business surveys, or like the Beveridge curve.

Thus, quantity constraints play an important role in macroeconomic analysis. However, constructing macroeconomic models with explicit aggregation over micromarkets was difficult, before the analysis was facilitated by Lambert (1988). Following early work and suggestions by Muellbauer (1978) and Malinvaud (1980), Lambert (1988) shows that aggregates of microeconomic minimums of demand and supply (output, employment) could be represented by a CES function of aggregate demand and supply. Moreover, the CES parameter $\rho$ reflects the degree of microeconomic dispersion, i.e., it can be interpreted as a parameter of mismatch. This result was later extended to the case with three components (to allow for capacity shortages; see Sneessens, 1983, and Sneessens-Drèze, 1986). The CES aggregation function served as a building block of the macroeconomic models used in the socalled European Unemployment Programme (see Drèze, Bean et al., 1990); the results obtained by econometric estimation on data from about ten countries proved the theoretical and empirical relevance of the CES aggregation for the understanding of Europe's unemployment problem.

Lambert's result and its extension to the three component case are based on the assumption that the micro-variables are jointly log-normally distributed; the CES function is then obtained as an approximation to the true aggregate function. In the mean time, Gourieroux et al. (1984) had shown that the CES function with two components can be interpreted as an 
exact result, provided one starts from Weibull rather than log-normal distributions. ${ }^{2}$ Building on this work, Heinesen (1993) has recently shown that the three component case can also be obtained as an exact result. The conditions needed for this result are, however, very restrictive. To obtain the simplest case where the CES three-component function has only one structural parameter, the micro-variables have to follow identical and independent Weibull distributions; the structural parameter appearing in the CES function is tight to the skewness of the Weibull distribution, which may be too restrictive to allow interesting economic interpretations. ${ }^{3}$ The two-stage (two-parameter) CES function can also be obtained as an exact result, but again under very restrictive, economically unappealing restrictions.

In this perspective, the interpretation of the CES function as an approximation rather than an exact result remains potentially very useful. By simply computing the approximation errors in different instances (which can easily be done by simulation experiments), one can check to what extent the CES function remains a useful tool of analysis when the variables do not satisfy the restrictive assumptions imposed by the exact approach based on Weibull distributions.

Smolny (1993) has analyzed the quality of the CES approximation in the instance of two components and has found errors being always less then $0.25 \%$. In this paper, we find errors of the same order of magnitude for the CES three-component function with only one structural parameter, whereas in the general case, complex and non-linear relationships between the approximation errors and the structural parameters emerge.

The purpose of this paper is twofold. We first show (section 2) how Lambert's result can be extended to the case with three components; we also derive the conditions required to obtain a CES function with one single structural parameter. In the most general case, no restriction needs to be imposed on the variance-covariance matrix. In section 3, we examine the quality of this approximation by the means of simulation experiments. It is shown that in realistic cases, the approximation error usually remains fairly small (around $1 \%$ or $2 \%$ ). We conclude with a few remarks in section 4.

\section{The CES-Approximation}

We start with a two-variable model. It is used to introduce the notation and the main intuitions. We next generalize to the three variable case and develop the CES approximation.

\subsection{The two-variable case}

There exits a continuum of micromarkets indexed by $i$ on the $[0,1]$ interval. Let us assume that on each micromarket, transacted quantities are determined by the minimum of demand and supply. To fix ideas, we will have in mind the example of the labour market, where real wage rigidities (not modelled here) are assumed to prevent the realization of a fullemployment equilibrium on each micromarket. In this context, employment $l$ on each micromarket is determined as the minimum of demand $x$ and supply $y$, i.e.:

\footnotetext{
2 For a simpler proof, see Heinesen (1995).

3 This result is slightly generalized in Heinesen (1994).
} 


$$
l_{i}=\min \left(x_{i}, y_{i}\right)
$$

We assume that the distribution of the $x_{i}{ }^{\prime}$ s and $y_{i}{ }^{\prime}$ s across micromarkets are generated by a log-normal process. More formally:

$$
\begin{array}{lrl}
x_{i}=\exp \left(\bar{x}+u_{i}\right), & \text { with } E(x)=\bar{X}=\exp \left(\bar{x}+\frac{1}{2} \sigma_{u}^{2}\right) \\
y_{i}=\exp \left(\bar{y}+v_{i}\right), & \text { with } E(y)=\bar{Y}=\exp \left(\bar{y}+\frac{1}{2} \sigma_{v}^{2}\right) \\
\text { where: } & (u, v) \sim N(0, \Sigma)
\end{array}
$$

$\bar{X}$ and $\bar{Y}$ correspond to aggregate demand and aggregate supply respectively. Aggregate employment $L$ is then obtained by explicit aggregation over micromarkets, i.e.,:

$$
L=E\left[\min \left(x_{i}, y_{i}\right)\right]=\int_{0}^{\infty} \int_{x}^{\infty} x f(x, y) d y d x+\int_{0}^{\infty} \int_{y}^{\infty} y f(x, y) d x d y .
$$

Standard manipulations involving changes of variables lead to the following expression:

$$
L=\bar{X} F\left(x^{*}-\frac{1}{2} \sigma^{*}\right)+\bar{Y} F\left(-x^{*}-\frac{1}{2} \sigma^{*}\right) \equiv \bar{X} F_{1}+\bar{Y} F_{2} \leq \min (\bar{X}, \bar{Y}),
$$

where $F($.$) is the cumulative standard normal distribution with arguments defined by:$

$$
\sigma^{*}=\sigma_{u}^{2}+\sigma_{v}^{2}-2 \sigma_{u v}, x^{*}=\frac{1}{\sigma^{*}} \ln \left(\frac{\bar{Y}}{\bar{X}}\right) .
$$

$\sigma^{*}$ is the variance of $u-v$. Because of the imperfect matching of demands and supplies at the micro level, aggregate employment is smaller than the minimum of aggregate demand and aggregate supply. It is worth stressing that, at given values of $\bar{X}$ and $\bar{Y}^{4}$, any change in the values of $\sigma_{u}^{2}, \sigma_{v}^{2}$ and $\sigma_{u v}$ that leaves the value of $\sigma^{*}$ unchanged has no effect on aggregate employment. In other words, the values of the variances and the covariance of the random terms $u$ and $v$ cannot be separately identified ${ }^{5}$. Hence that writing the variance covariance matrix $\sum$ as

$$
\sum=\sigma^{2}\left[\begin{array}{ll}
1 & r \\
r & 1
\end{array}\right]
$$

${ }^{4}$ Remember that the value of $\bar{X}$ and $\bar{Y}$ depends on $\sigma_{u}^{2}$ and $\sigma_{v}^{2}$ respectively.

5 To get the intuition, let us consider the simpler case with normal variates, i.e.: $l_{i}=\min \left(\bar{x}+u_{i}, \bar{y}+v_{i}\right)$. Then

$$
l_{i}= \begin{cases}\bar{x}+u_{i} & \text { if } u_{i}-v_{i} \leq \bar{y}-\bar{x} \\ \bar{y}+v_{i} & \text { otherwise }\end{cases}
$$

which illustrates why it is the variance of $(u-v)$ that matters. 
entails no loss of generality. With this notation, the variance of $(u-v)$ is given by:

$$
\sigma^{2}=2 \sigma^{2}(1-r)
$$

which means that there is still one parameter "too much" ( $\sigma$ and $r$ cannot be separately identified). This notation shows that the correlation between $u$ and $v$ is crucial: if $r$ goes to 1 , $\sigma^{*}$ goes to zero and there is no heterogeneity across micro markets (no "mismatch"), whatever the values of $\sigma_{u}^{2}$ and $\sigma_{v}^{2}$. In this extreme case, the expression determining aggregate employment is simply the minimum of aggregate demand and aggregate supply.

\subsection{Generalization to three variables ${ }^{6}$}

The previous setup can be extended to have three rather than two variables. Such an extension may be desirable in several instances. One may wish for instance to distinguish different types of labour (skilled and unskilled) and leave open the possibility of both skilled and unskilled labour shortages; or one may wish to allow for capacity constraints. Other examples are certainly possible. In the rest of the paper, we will rely, to explain the notation and give the intuitions, on the capacity constraint example.

On each micromarket, employment is determined either by the demand for goods (sales constraint) ${ }^{7}$, or by the capacity constraint (capacity-employment) or by the supply of labour. Assuming again the distribution across micromarkets follows a log-normal process, we will write these three determinants as:

$$
\begin{array}{lll}
\text { demand-determ.empl.: } & x_{i}=\exp \left(\bar{x}+u_{i}\right), & E(x)=\bar{X}=\exp \left(\bar{x}+\frac{1}{2} \sigma_{u}^{2}\right) \\
\text { capacity-determ.empl.: } & y_{i}=\exp \left(\bar{y}+v_{i}\right), & E(y)=\bar{Y}=\exp \left(\bar{y}+\frac{1}{2} \sigma_{v}^{2}\right) \\
\text { lab.supply-determ.empl.: } & z_{i}=\exp \left(\bar{z}+w_{i}\right), & E(z)=\bar{Z}=\exp \left(\bar{z}+\frac{1}{2} \sigma_{w}^{2}\right)
\end{array}
$$

Employment on each micromarket is the minimum of these three quantities:

$$
l_{i}=\min \left(x_{i}, y_{i}, z_{i}\right)=\min \left(\bar{x}+u_{i}, \bar{y}+v_{i}, \bar{z}+w_{i}\right)
$$

where $(u, v, w) \sim N(0, \Sigma)$.

Aggregate employment $L$ is obtained by explicit aggregation over micromarkets, i.e.:

$$
L=E\left[\min \left(x_{i}, y_{i}, z_{i}\right)\right]=L_{1}+L_{2}+L_{3}
$$

\footnotetext{
6 This section is based on Sneessens (1983).

7 The demand-determined employment level is often referred to as the Keynesian demand for labour, which may be a misleading terminology when, in a monopolistic competition setup, the "constraint" actually results from the price behaviour of the firm rather than a nominal rigidity.
} 
where:

$$
L_{1}=\int_{0}^{\infty} \int_{x}^{\infty} \int_{x}^{\infty} x f(x, y, z) d z d y d x, \quad L_{2}=\int_{0}^{\infty} \int_{y}^{\infty} \int_{y}^{\infty} y f(x, y, z) d z d x d y
$$

$$
L_{3}=\int_{0}^{\infty} \int_{z}^{\infty} \int_{z}^{\infty} z f(x, y, z) d y d x d z
$$

As in the two-variable case, standard (tedious) manipulations involving several changes of variables eventually lead to the following expression (see appendix):

$$
L=\bar{X} F_{1}+\bar{Y} F_{2}+\bar{Z} F_{3}
$$

where:

$$
\begin{aligned}
& F_{1} \equiv F\left(x_{2}^{*}-\frac{1}{2} \sigma_{2}^{*}, \quad x_{3}^{*}-\frac{1}{2} \sigma_{3}^{*} \mid \tilde{\rho}_{1}\right), \quad F_{2} \equiv F\left(-x_{2}^{*}-\frac{1}{2} \sigma_{2}^{*}, \quad y_{3}^{*}-\frac{1}{2} \sigma_{4}^{*} \mid \tilde{\rho}_{2}\right), \\
& F_{3} \equiv F\left(-x_{3}^{*}-\frac{1}{2} \sigma_{3}^{*}, \quad-y_{3}^{*}-\frac{1}{2} \sigma_{4}^{*} \mid \tilde{\rho}_{3}\right),
\end{aligned}
$$

where the $F_{i}$ 's stand for the bivariate cumulative standard normal distribution function $F\left(. . . \tilde{\rho}_{i}\right)$ where $\tilde{\rho}_{i}$ is the correlation between the standard normal variables. The arguments of the function are defined by:

$$
\begin{aligned}
& \sigma_{2}^{* 2}=\operatorname{var}(u-v)=\operatorname{var}(\ln (x / y))=\sigma_{u}^{2}+\sigma_{v}^{2}-2 \sigma_{u v}, \\
& \sigma_{3}^{* 2}=\operatorname{var}(u-w)=\operatorname{var}(\ln (x / z))=\sigma_{u}^{2}+\sigma_{w}^{2}-2 \sigma_{u w}, \\
& \sigma_{4}^{* 2}=\operatorname{var}(v-w)=\operatorname{var}(\ln (y / z))=\sigma_{v}^{2}+\sigma_{w}^{2}-2 \sigma_{v w}, \\
& x_{2}^{*} \equiv \frac{1}{\sigma_{2}^{*}} \ln \left(\frac{\bar{Y}}{\bar{X}}\right), \quad x_{3}^{*} \equiv \frac{1}{\sigma_{3}^{*}} \ln \left(\frac{\bar{Z}}{\bar{X}}\right), \quad y_{3}^{*} \equiv \frac{1}{\sigma_{4}^{*}} \ln \left(\frac{\bar{Z}}{\bar{Y}}\right) .
\end{aligned}
$$

For further details and a proof see appendix. Remember that $\bar{X}, \bar{Y}, \bar{Z}$ are across-market averages of $x, y, z$ respectively. The comments made about the two-variable model apply here as well, mutatis mutandis. The elements of the original variance-covariance matrix cannot be separately identified; only the three combinations $\sigma_{2}^{*} \quad$ (variance of $u-v$ ), $\sigma_{3}^{*}$ (variance of $u-w$ ) and $\sigma_{4}^{*}$ (variance of $v-w$ ) are identifiable. All the heterogeneity across micromarkets (mismatch) is summarized in these three parameters. As a result, writing the variance-covariance matrix in the specific form

$$
\sum=\sigma^{2}\left[\begin{array}{ccc}
1 & r_{12} & r_{13} \\
& 1 & r_{23} \\
& & 1
\end{array}\right]
$$


entails no loss of generality. With this notation, the variances of $u-v, u-w$ and $v-w$ are given respectively by:

$$
\sigma_{2}^{*^{2}}=2 \sigma^{2}\left(1-r_{12}\right), \quad \sigma_{3}^{*^{3}}=2 \sigma^{2}\left(1-r_{13}\right), \quad \sigma_{4}^{*^{2}}=2 \sigma^{2}\left(1-r_{23}\right)
$$

which means that there is still one parameter "too much" ( $\sigma$ and the three correlation coefficients $r_{j}$ cannot be separately identified: only the three $\sigma_{i}^{*^{2}}$ 's are econometrically identifiable). Still, this notation is convenient because it helps to distinguish between the size of the idiosyncratic shocks and their correlation structure. ${ }^{8}$

\subsection{Regime Proportions}

It can be shown that the weighted proportion of micromarkets in a particular regime (see Lambert (1988) for a definition) is equal to the ratio between the contribution of that particular regime to aggregate employment (see equation (2.10)) and aggregate employment itself. For instance, the weighted proportion of micromarkets in the first regime, defined by:

$$
l_{i}=x_{i}=\min \left(x_{i}, y_{i}, z_{i}\right)
$$

is given by the formula:

$$
P_{1}=\frac{\bar{X} F_{1}}{L}=\frac{\bar{X} F_{1}}{\bar{X} F_{1}+\bar{Y} F_{2}+\bar{Z} F_{3}}=\left(1+\frac{\bar{Y}}{\bar{X}} \frac{F_{2}}{F_{1}}+\frac{\bar{Z}}{\bar{X}} \frac{F_{3}}{F_{1}}\right)^{-1} \leq 1
$$

One obtains similarly $P_{2}=\bar{Y} F_{2} / L$ and $P_{3}=\bar{Z} F_{3} / L$, which, together with (2.10) always implies $P_{1}+P_{2}+P_{3}=1$ as expected. Data are often available on $P_{1}, P_{2}$ and $P_{3}$, while none are available for $F_{1}, F_{2}, F_{3}$. Hence the estimation of relationships like (2.10)-(2.13) is far from trivial. One possibility is to approximate the double integrals embedded in the $F_{i}$ 's by easier analytical expressions.

\subsection{Approximating the $F_{i}^{\prime} \mathrm{s}$}

Many alternatives are open. One can for example approximate the normal distribution function by a Sargan distribution function or by a logistic function. In our context, it is perhaps more natural to approximate $F_{i}$ by $P_{i}$ itself. By using (2.11) and (2.12), it can be seen that both $F_{i}$ and $P_{i}$ can be written as functions of $\bar{X}, \bar{Y}$ and $\bar{Z}$.

One can then easily check the following properties: (i) the derivatives of $F_{i}$ and $P_{i}$ with respect to $\bar{X}, \bar{Y}$ and $\bar{Z}$ have the same signs; (ii) the limits of $F_{i}$ and $P_{i}$ for $\bar{X}, \bar{Y}$ and $\bar{Z}$ going to zero or plus infinity are identical. These properties suggest a situation like the one depicted in Figure 1 for the particular case $i=1$ and $\bar{X}=\bar{Z}$.In

8 One should also remember that changing the values of, say, $\sigma_{u}^{2}$ changes the value of $\bar{X}$. 
Figure 1: The approximation of $F_{1}$

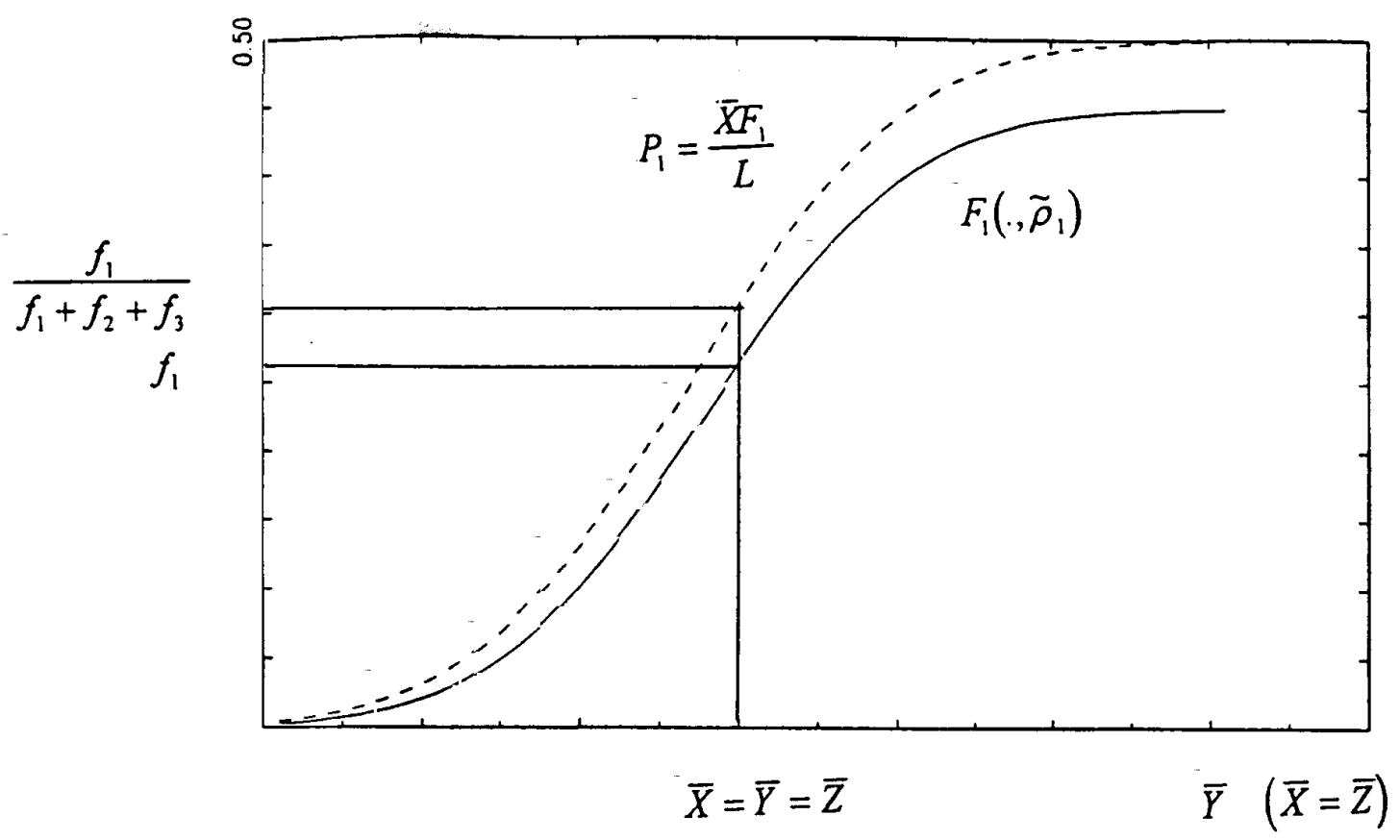

practice, it will probably be true in most cases that $\bar{X}, \bar{Y}$ and $\bar{Z}$ are not too far apart; that is, most values of $F_{i}, P_{i}$ will probably take place around the particular values:

$$
f_{i}=F_{i}\left(0-\frac{1}{2} \sigma_{m}^{*}, 0-\frac{1}{2} \sigma_{n}^{*} \mid \tilde{\rho}_{i}\right) \quad p_{i}=\frac{f_{i}}{f_{1}+f_{2}+f_{3}}
$$

One may thus want the approximation of $F_{i}$ by $P_{i}$ to be correct at that point. This is achieved by using 9

$$
F_{i} \simeq P_{i}^{\left(1+\rho_{i}\right) / \rho_{i}} \quad \rho_{\mathrm{i}} \geq 0
$$

where

$$
\rho_{i}=\frac{\ln p_{i}}{\ln \left(f_{1}+f_{2}+f_{3}\right)}
$$

In that way, one obtains a correct approximation at the point $\bar{X}=\bar{Y}=\bar{Z}$ without losing the two sets of properties listed above.

9 Bierings-Muysken (1988) have suggested an alternative approximation formula with:

$$
\begin{aligned}
& F_{1} \cong P_{1}^{1+\rho_{1} / \rho_{1}}, \text { and } \\
& F_{i} \cong P_{i}^{1+\rho_{1} / \rho_{1}} \cdot \frac{\left(P_{2}+P_{3}\right)^{1 / \rho_{1}}}{\left(P_{2}^{\rho_{2} / \rho_{1}}+P_{3}^{\rho_{2} / \rho_{1}}\right)^{1 / \rho_{1}}}, \quad i=2,3,
\end{aligned}
$$

which allows them to obtain two-stage (two-parameter) CES functions. The same two-stage CES function has been justified by Smolny (1993) on the ground that the minimum of two log-normally distributed variables is itself approximately log-normally distributed." 
Combining (2.13) and (2.14) implies:

$$
P_{1} \simeq\left(\frac{\bar{X}}{L}\right)^{-\rho_{1}}
$$

which in turn implies via equations (2.10) and (2.14):

$$
\left(\frac{\bar{X}}{L}\right)^{-\rho_{1}}+\left(\frac{\bar{Y}}{L}\right)^{-\rho_{2}}+\left(\frac{\bar{Z}}{L}\right)^{-\rho_{3}} \approx 1
$$

There are three "mismatch" parameters: $\rho_{1}, \rho_{2}$ and $\rho_{3}$, corresponding to the variances $\sigma_{1}^{*}, \sigma_{2}^{*}$ and $\sigma_{3}^{*}$. In the particular case where $\sigma_{2}^{*}=\sigma_{3}^{*}=\sigma_{4}^{*}=\sigma^{*}$, the pairwise correlation coefficients between $u, v$ and $w$ are all equal $: r_{12}=r_{13}=r_{23}=r$, which is sufficient to imply that $f_{1}=f_{2}=f_{3}$. In this particular case, all $\rho$ 's are equal: $\rho_{1}=\rho_{2}=\rho_{3}=\rho$, and the approximation (2.16) giving aggregate employment boils down to a CES function with one single approximation coefficient (corresponding to the single mismatch parameter $\sigma^{*}$ ):

$$
L \simeq\left[\bar{X}^{-\rho}+\bar{Y}^{-\rho}+\bar{Z}^{-\rho}\right]^{-1 / \rho}
$$

\subsection{Remark}

In the two-variable case, $f_{1}=f_{2}=F\left(-\sigma^{*} / 2\right)$, which implies $p_{1}=p_{2}=0.5$ and $\rho_{1}=\rho_{2}=\rho$. In other words, in the two-variable case, there is single approximation coefficient (corresponding to the single mismatch parameter $\sigma^{*}$ ) and the approximate transaction (employment) function is always in the form of an explicit CES function with one parameter $\rho$. The final result is thus similar to that in Lambert (1988) though ist derivation and the definition of $\rho$ is different:

$$
\rho_{J P L}=-1+\frac{2 f\left(-\sigma^{*} / 2\right)}{\sigma^{*} F\left(-\sigma^{*} / 2\right)}, \quad \rho_{H S}=-1+\frac{\ln F\left(-\sigma^{*} / 2\right)}{\ln 2+\ln F\left(-\sigma^{*} / 2\right)}
$$

where $f$ is the standard normal density. 


\section{Approximation Errors: Numerical Examples}

\subsection{Design of the Experiment}

We consider micromarkets with three components $x_{i}, y_{i}$ and $z_{i}$ :

$$
\begin{aligned}
& \ln x_{i}=\bar{x}+u_{i}, \\
& \ln y_{i}=\bar{y}+v_{i}, \\
& \ln z_{i}=\bar{z}+w_{i},
\end{aligned}
$$

where $(u, v, w) \sim N(0, \Sigma)$. Defining the expected values of the components as $\bar{X}, \bar{Y}$ and $\bar{Z}$, our analysis starts with the computation of the expected value of the minimum condition (see (2.10))

$$
L=E\left(\min \left(X_{i}, Y_{i}, Z_{i}\right)\right)=\bar{X} F_{1}+\bar{Y} F_{2}+\bar{Z} F_{3}
$$

where $F_{1}, F_{2}$ and $F_{3}$ are calculated according to (2.11), and by applying the GAUSS function CDFBVN (bivariate standard normal cumulative distribution function).

Below, the true expected value (3.1) will be compared with its CES approximation. We use the approximation (2.16) instead of the usual CES function (2.17) because it is more general and it can be extended to the analysis of situations with three different values $\rho_{1}, \rho_{2}, \rho_{3}$. The approximation errors are reported as

$$
\text { error }=S-1,
$$

where we define the sum of regime proportions

$$
S=\left(\frac{\bar{X}}{L}\right)^{-\rho_{1}}+\left(\frac{\bar{Y}}{L}\right)^{-\rho_{2}}+\left(\frac{\bar{Z}}{L}\right)^{-\rho_{3}}
$$

according to (2.16).

It should be stressed that this approach represents a conservative test. Using the approximation (2.17)

$$
\tilde{L}=\left(\bar{X}^{-\rho}+\bar{Y}^{-\rho}+\bar{Z}^{-\rho}\right)^{(-1 / \rho)}
$$

the error would be much closer to zero. This can be seen by calculating

$$
\tilde{L}^{-\rho}=L^{-\rho} S=\bar{Y}^{-\rho}+\bar{X}^{-\rho}+\bar{Z}^{-\rho},
$$

implying

$$
\tilde{L}=L \cdot S^{-1 / \rho}
$$

Defining the proportional error as "error $1 "=(L-\tilde{L}) / L$, we obtain

$$
\text { error } 1=1-S^{-1 / \rho}
$$


With $\rho$ ranging between 15 and $60^{10}$, the error in terms of employment becomes much smaller, at least by a factor 10 . For example, $S=1.05, \rho=15$ yields "error 1 " $=0.003$ instead of "error" $=0.05$.

We define the variance-covariance matrix as

$$
\Sigma=\sigma^{2} R, \quad R=\left(\begin{array}{ccc}
1 & r_{12} & r_{13} \\
r_{12} & 1 & r_{23} \\
r_{13} & r_{23} & 1
\end{array}\right) .
$$

$r_{12}, r_{13}$ and $r_{23}$ are the correlation coefficients between the components $\ln X_{i}$, $\ln Y_{i}$ and $\ln Z_{i}$.

Let us denote our control variable by $P$ (think of demand and supply depending on prices). Defining $\bar{x} \equiv P$ and $\bar{y} \equiv \delta-P$, we may write:

$$
\begin{aligned}
& \ln X_{i}=P+u_{i} \\
& \ln Y_{i}=\delta-P+v_{i} \\
& \ln Z_{i}=\bar{z}+w_{i} .
\end{aligned}
$$

Then, varying $P$ according to $P=0.8,0.82, \ldots, 0.98,1.00,1.02, \ldots, 1.18,1.20$ (i.e. $P=0.8$ $+(\alpha-1) 0.02, \alpha=1, \ldots, 21)$ and assuming $\delta=2$, we cover 21 different situations symmetric to the equality of $\bar{X}$ and $\bar{Y}$ at $P=\bar{x}=\bar{y}=1$. The ratio of expected values, $\bar{X} / \bar{Y}=\exp (\bar{x}-\bar{y})$ (for $\sigma_{u}^{2}=\sigma_{v}^{2}$; see (3.1)) ranges from 0.67 to 1.49 . It is important to note that the accuracy of the approximation depends on these ratios only, and not on levels, as can be seen by looking at the equations for $F_{i}, P_{i}, i=1,2,3$, and $L$ (cfr equations (2.10)-(2.13)). Hence, presented results do cover general situations and not just special cases. To fully determine the simulation experiments, it remains to vary $\bar{z}$ and fix the values of the variancecovariance matrix. We first consider the case with identical correlation coefficients between pairs of variables.

\subsection{Assuming Identical Correlation Coefficients}

In the following, in a first group of experiments, we assume

$$
\Sigma=\sigma^{2}\left(\begin{array}{lll}
1 & r & r \\
r & 1 & r \\
r & r & 1
\end{array}\right)
$$

i.e. the variances of $(\log ) x, y$, and $z$ must be equal and the pairwise correlations must also be equal. On these conditions, we have

$$
\sigma_{i}^{* 2}=2 \sigma^{2}(1-r), \quad i=2,3,4
$$

10 See the work of the "European Unemployment Programme" (Drèze et al., 1990) where most estimates of SURE ["structural unemployment at equilibrium", see also below], which are based on $\rho$, range between $1 \%$ and $7 \%$. 
In the limiting case $r \rightarrow 1$ the mismatch disappears (perfect proportionality of $x, y$ and $z$ across micro markets) and both (2.10) and (2.17) converge to the aggregate min-condition.

Figures 2 to 4 illustrate how "error" depends on the parameters $\bar{x}, \bar{y}, \bar{z}, \sigma$ and $r$. The value of the control variable $P$ (which determines the ratio $\bar{X} / \bar{Y}$ ) is measured along the horizontal axis. Each figure corresponds to a different value of the correlation coefficient $r(r=0.00$, $0.50,0.90)$; each is made of six panels, corresponding to different values for $\bar{z}(\bar{z}=0.50,0.80$, $0.95,1.00,1.05,1.20$ respectively). We obtain in this way situations below, at, and above the general equilibrium $\bar{x}=\bar{y}=\bar{z}=1$. Each panel furthermore reproduces the approximation error for four different values of the variance $\sigma^{2}\left(\sigma^{2}=0.005,0.01,0.02,0.04\right)$. $\sigma$ and $r$ determine $\sigma_{\mathrm{i}}{ }^{*}, i=2,3,4$, which in turn determines $\rho$ (see (2.14)). $\rho$ can be more economically expressed in terms of SURE ("structural underutilization rate at equality", see Sneessens and Drèze, 1986). Considering the situation of a hypothetical equilibrium $\bar{X}=\bar{Y}=\bar{Z}$, which leads to $L=3^{-1 / \rho} \bar{X}$, and defining the rate of underutilization, $U R$, as $U R=1-L / \bar{X}$, we obtain

$$
S U R E=1-3^{-1 / \rho}
$$

Increasing correlation gives increasing $\rho$ and, hence, a decreasing dispersion on micromarkets, i.e. a decreasing SURE. For instance, $\sigma^{2}=0.005,0.01,0.02,0.04$ and $r=0$ lead to $S U R E=0.059,0.083,0.117,0.163$, respectively (see also Figure 2), whereas the same variances and $r=0.9$ lead to $S U R E=0.012,0.027,0.038,0.053$ (confer also Figure 4). ${ }^{11}$

Before we discuss the figures more closely, it is worth summarizing the results by computing average errors over all $P^{\prime} s$ and $\bar{z} ' s$. This is done in Table 1 . The approximation errors are very small. Recall that "error" gives the discrepancy between the sum of approximate regime proportions and its true value 1. "Error\%" is defined as "error" * 100. "Error 1\%", defined as "errorl" * 100, gives the approximation error in percent of actual employment L. The average values range from a minimum of $0.002 \%$ to a maximum of $0.12 \%$ (see Table 1a). The maximal error over all replications (for the unrealistic case of $S U R E \%=16,3 \%$, based on $r=0$ and $\sigma^{2}=0.04$ ) is equal to $0.41 \%$ (see Table $1 b$ ).

The figures give a more detailed information on "error". Figure 2 describes zero correlation, Figure 3 and Figure 4 assume $r=0.5$ and $r=0.9$, respectively. The graphics reveal a highly nonlinear relationship between the parameters under consideration. Most important, however, is the very low level of approximation errors which remain below $0.04(4 \%)$ even in the worst case. ${ }^{12}$ There are too many cases to comment all of them in detail, but some general conclusions can be drawn. Starting with the situation of equilibrium $(\bar{z}=1)$, we first confirm the theoretical result of a correct approximation at equilibrium (see Section 2). Moving away from the equilibrium (considering $P<1, P>1$ ), we observe first growing and then decreasing errors. If $r$ is high enough (compare Figures 2-4), we can observe a recurrence towards zero approximation errors even in the close neighbourhood of the general equilibrium $\bar{x}=\bar{y}=\bar{z}$. Likewise, the range of almost zero approximation errors is getting smaller when $\sigma$ (SURE) decreases.

\footnotetext{
${ }^{11}$ Note from (3.7) that changing $(1-r)$ or changing $\sigma^{2}$ may give the same result. Such cases are covered in Table 1 with $r=0$ and $r=0.5$.

${ }^{12}$ Remember that in terms of levels approximation errors are much smaller (see Table 1).
} 
If $\bar{z}>1$, the error is getting negative at $\bar{x}=\bar{y}=1$, being about -0.04 at its minimum. For the other regions, we observe the same result as before: when either the correlation coefficient is high and/or the variance is low, the error approaches zero for $P>1, P<1$. Things are less obvious for $\bar{z}<1$.In this case, at the equilibrium, we find both, errors approaching zero and moving away from zero. The direction depends on $r, \sigma$, and the proximity to the general equilibrium $\bar{x}=\bar{y}=\bar{z}=1$. For instance, at $\bar{z}=0.8$, we see that the curve is bending downward (approaching zero) when either $\sigma$ is small and / or $r$ is high enough (see Figures 3 and 4).

\section{Table 1: Summary of Approximation Errors}

a) Average values of absolute errors: $\mid$ error $\%|/|$ error $1 \% \mid$

\begin{tabular}{ccccc} 
& $r=0$ & $r=0.5$ & $r=0.9$ & $r=0.98$ \\
\hline$\sigma^{2}=0.005$ & $1.00 / 0.055$ & $0.85 / 0.033$ & $0.48 / 0.008$ & $0.27 / 0.002$ \\
& $(5.9)$ & $(4.2)$ & $(1.2)$ & $(0.8)$ \\
$\sigma^{2}=0.01$ & $1.06 / 0.084$ & $1.00 / 0.055$ & $0.62 / 0.015$ & $0.36 / 0.004$ \\
& $(8.3)$ & $(5.9)$ & $(2.7)$ & $(1.2)$ \\
$\sigma^{2}=0.02$ & $1.00 / 0.112$ & $1.06 / 0.084$ & $0.79 / 0.07$ & $0.45 / 0.007$ \\
& $(11.7)$ & $(8.3)$ & $(3.8)$ & $(1.7)$ \\
$\sigma^{2}=0.04$ & $0.77 / 0.123$ & $1.00 / 0.112$ & $0.95 / 0.047$ & $0.57 / 0.013$ \\
& $(16.3)$ & $(11.7)$ & $(5.3)$ & $(2.4)$ \\
\hline
\end{tabular}

b) Maximal absolute errors: $\max (\mid$ error $\% \mid) / \max (\mid$ error $1 \% \mid)$

\begin{tabular}{ccccc} 
& $r=0$ & $r=0.5$ & $r=0.9$ & $r=0.98$ \\
\hline$\sigma^{2}=0.005$ & $3.9 / 0.22$ & $3.9 / 0.16$ & $3.9 / 0.07$ & $3.9 / 0.03$ \\
& $(5.9)$ & $(4.2)$ & $(1.2)$ & $(0.8)$ \\
$\sigma^{2}=0.01$ & $3.7 / 0.3$ & $3.9 / 0.22$ & $3.9 / 0.1$ & $3.9 / 0.04$ \\
& $(8.3)$ & $(5.9)$ & $(2.7)$ & $(1.2)$ \\
$\sigma^{2}=0.02$ & $2.9 / 0.33$ & $3.7 / 0.3$ & $3.9 / 0.14$ & $3.9 / 0.06$ \\
& $(11.7)$ & $(8.3)$ & $(3.8)$ & $(1.7)$ \\
$\sigma^{2}=0.04$ & $2.5 / 0.41$ & $2.9 / 0.33$ & $3.9 / 0.19$ & $3.9 / 0.09$ \\
& $(16.3)$ & $(11.7)$ & $(5.3)$ & $(2.4)$ \\
\hline
\end{tabular}

Note: Averages and extreme values according to equations (3.2) and (3.4) measured in percentage points; for each $r$ and $\sigma$, we average over all $P$ and $\bar{z}$. Upper value: "error\%"/"error $1 \% "$, in parentheses: SURE \%. 
Figure 2: Approximation errors: Values of "error" for $r=0$.
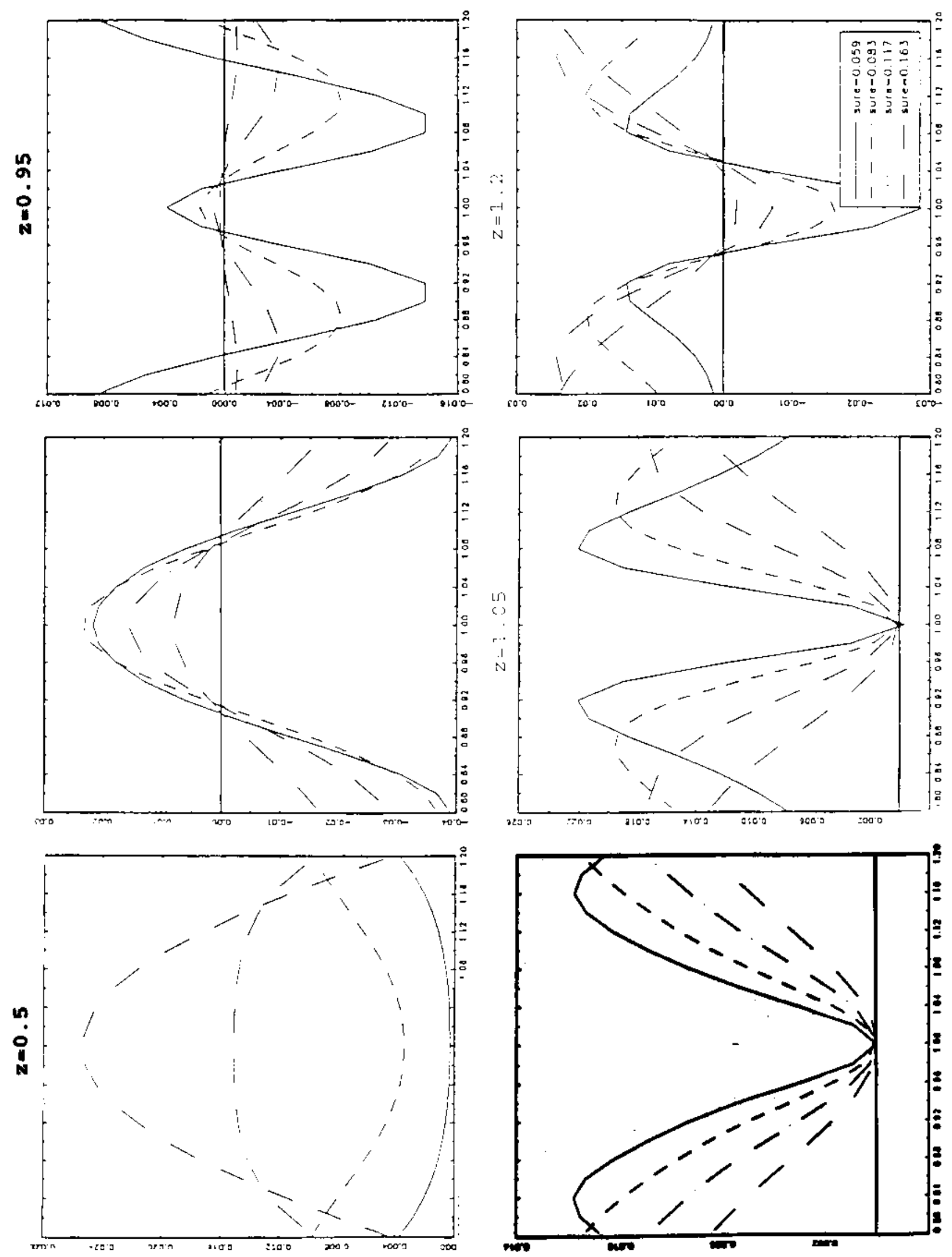
Figure 3: Approximation errors: Values of "error" for $r=0.5$.
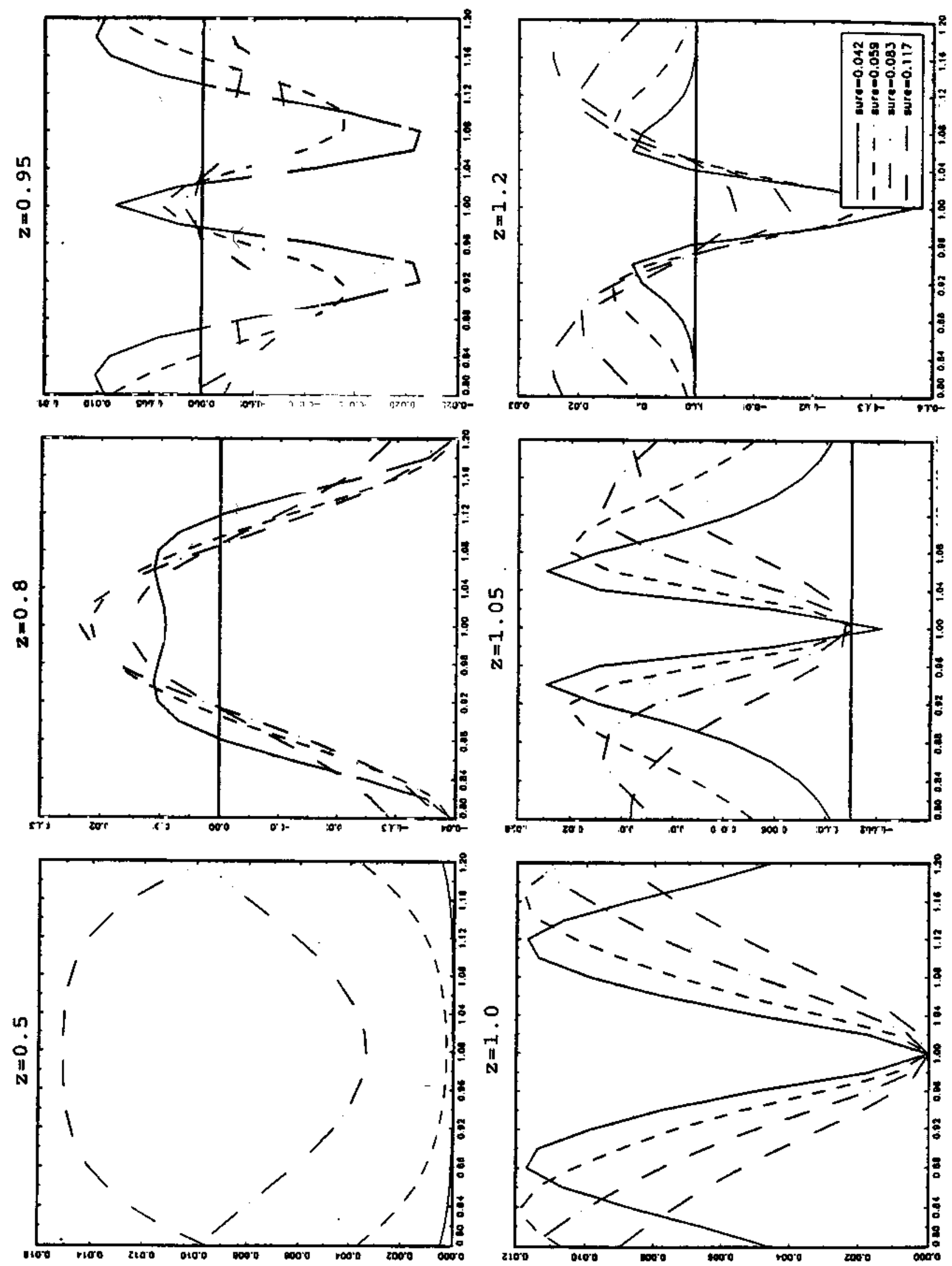
Figure 4: Approximation errors: Values of "error" for $r=0.9$.
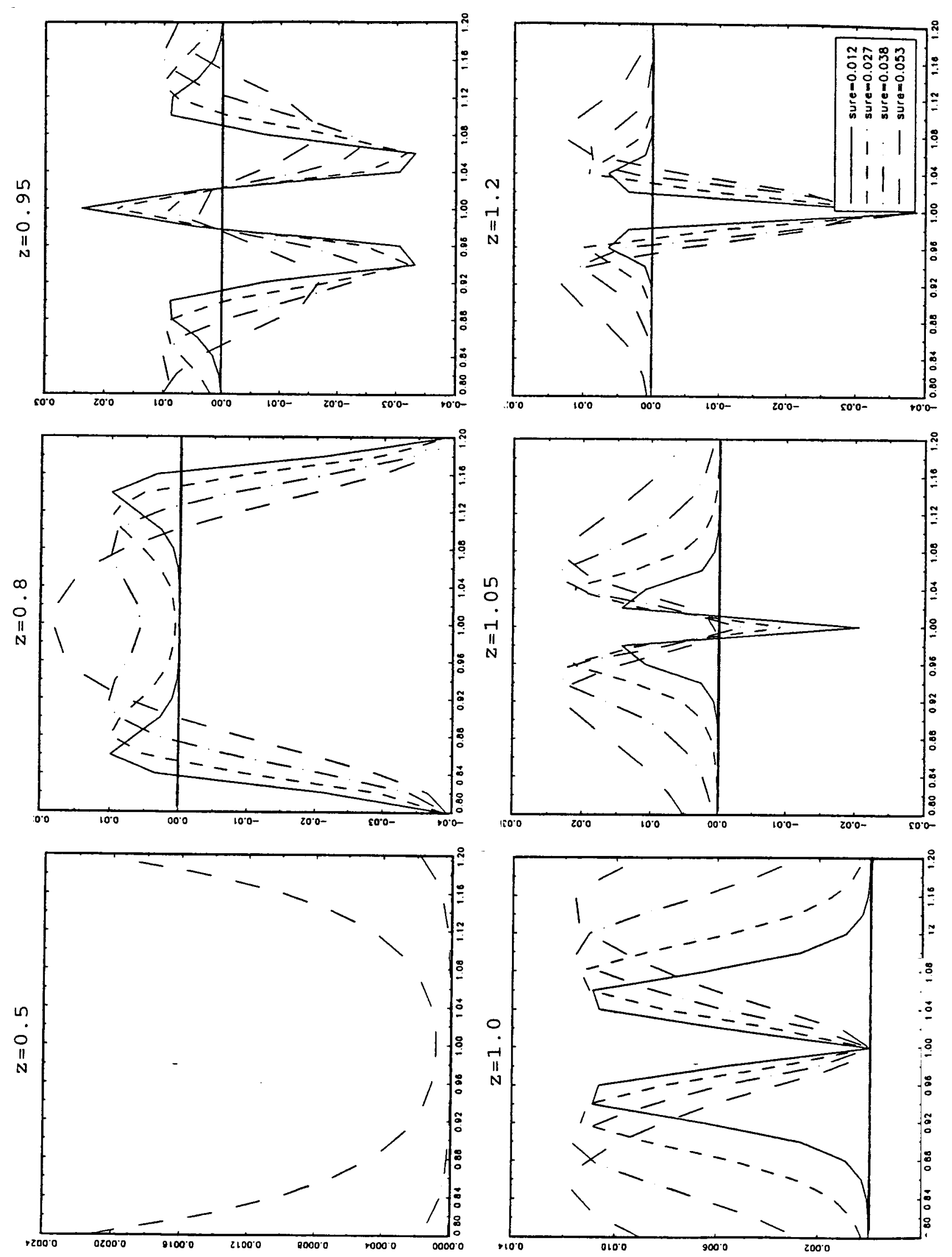


\subsection{The Approximation Bias in the Case of Different Correlation Coefficients}

Up to now we assumed identical correlation coefficients and relied on the CES approximation formula. Equation (2.16) allows one to consider cases with more general variance-covariance structure, by introducing three different parameters, $\rho_{1}, \rho_{2}, \rho_{3}$. We now repeat our simulation experiments in this more general setup. Our experiments are designed in the same way as before (see Section 3.1). Without loosing too much information, we restrict ourselves to the case $\bar{z}=1$, but we vary $P$ (measured along the horizontal axis) as before. Thus, we still consider situations in equilibrium and disequilibrium.

As before, approximation errors are measured by "error\%" and "error $1 \% "$ ". Since there are different $\rho_{i}$, no explicit solution of $\tilde{L}$ exists. Instead, we present "error1\%" using implicit numerical approximate solutions of equation (2.16). Thus, we find $\tilde{L}$ as

$$
\tilde{L}=\arg \min _{L^{*}}\left|1-\left(\frac{\bar{X}}{L^{*}}\right)^{-\rho_{1}}-\left(\frac{\bar{Y}}{L^{*}}\right)^{-\rho_{2}}-\left(\frac{\bar{Z}}{L^{*}}\right)^{-\rho_{3}}\right| .
$$

With respect to the correlation between the three components, we distinguish three different situations. First, a situation with low and relatively homogeneous correlation coefficients, all being less than 0.5 , second, a situation with high and relatively homogeneous correlation coefficients, all being larger than 0.5 , and, finally, a situation with heterogeneous correlation coefficients.

The more general $\Sigma$ requires a new way to calculate $S U R E$ : Using the result

$$
L^{*} \equiv E\left(\min \left(X_{i}, Y_{i}, Z_{i}\right) \mid \bar{X}=\bar{Y}=\bar{Z}\right)=\bar{X}\left(F_{1}+F_{2}+F_{3}\right)
$$

of equilibrium employment, $S U R E$ can be computed as

$$
S U R E=1-\frac{L^{*}}{\bar{X}}=1-F_{1}-F_{2}-F_{3}
$$

where $F_{i}, \quad i=1,2,3$, are defined according to (2.11).

In the following, we consider three experiments using the following variance-covariance matrices:

$$
R_{1}=\left(\begin{array}{ccc}
1 & 0.4 & 0.2 \\
0.4 & 1 & 0.1 \\
0.2 & 0.1 & 1
\end{array}\right), \quad R_{2}=\left(\begin{array}{ccc}
1 & 0.8 & 0.5 \\
0.8 & 1 & 0.6 \\
0.5 & 0.6 & 1
\end{array}\right), \quad R_{3}=\left(\begin{array}{ccc}
1 & 0.8 & 0.5 \\
0.8 & 1 & 0.25 \\
0.5 & 0.25 & 1
\end{array}\right)
$$


Given $R_{1}, \sigma^{2}=0.005,0.01,0.02,0.04$ implies $S U R E \%=5.2 \%, 7.3 \%, 10.2 \%$ and $14.3 \%$, respectively. Table 2.a) reveals that average errors are about $1 \%$ ("error\%"), resp. $0.1 \%$ ("error $1 \% ") . \rho_{1}, \rho_{2}$ and $\rho_{3}$ (computed as in (2.14)) do not differ much. ${ }^{13}$

Secondly, we assume $R_{2}$. Table 2.b) confirms the small errors found in Table 2.a). "error $1 \%$ " remains at the very low level and ranges between $0.03 \%$ and $0.2 \%$, "error\%" is about $2 \%$.

Table 3.c) reveals that the heterogenous case $R_{3}$ leads to somewhat higher errors than for homogenous correlation coefficients. Nevertheless, even in the worst case, with SURE being at the very high level of $11.2 \%$, "error\%" is $3.9 \%$. In term of levels, i.e. in term of "error $1 \%$ ", this would mean $0.5 \%$.

13 Preliminary estimates on Belgian data suggest that $\rho_{1}, \rho_{2}$ and $\rho_{3}$ are in fact not significantly different from one another. See Nilles (1991). 
Table 2: Summary of Approximation Errors: General Correlation Structure

$$
\sigma^{2}=0.005 \quad \sigma^{2}=0.01 \quad \sigma^{2}=0.02 \quad \sigma^{2}=0.04
$$

a) Small and homogenous correlation coefficients: $R_{1}$

$\begin{array}{lcccc}\rho_{1} & 22.2 & 15.6 & 10.9 & 7.6 \\ \rho_{2} & 20.9 & 14.7 & 10.3 & 7.2 \\ \rho_{3} & 19.1 & 13.4 & 9.4 & 6.5 \\ \text { SURE\% } & 5.2 & 7.3 & 10.2 & 14.3 \\ \text { mean of |error\%| } & 1.0 & 1.0 & 1.1 & 1.0 \\ \text { mean of |error } 1 \% \text { | } & 0.1 & 0.1 & 0.1 & 0.1\end{array}$

b) High and homogenous correlation coefficients: $R_{2}$

$\begin{array}{lcccc}\rho_{1} & 30.8 & 21.7 & 15.2 & 10.7 \\ \rho_{2} & 35.6 & 25.0 & 17.6 & 12.4 \\ \rho_{3} & 25.9 & 18.2 & 12.8 & 8.9 \\ \text { SURE\% } & 3.5 & 5.0 & 7.0 & 9.9 \\ \text { mean of |error\%| } & 1.4 & 1.7 & 2.0 & 2.2 \\ \text { mean of |error } 1 \% \text { | } & 0.03 & 0.1 & 0.1 & 0.2\end{array}$

c) Heterogenous correlation coefficients: $R_{3}$

$\begin{array}{lcccc}\rho_{1} & 35.4 & 24.9 & 17.6 & 12.3 \\ \rho_{2} & 25.7 & 18.1 & 12.6 & 8.8 \\ \rho_{3} & 21.5 & 15.1 & 10.6 & 7.4 \\ \text { SURE\% } & 4.0 & 5.7 & 8.0 & 11.2 \\ \text { mean of |error\%| } & 2.5 & 3.1 & 3.7 & 3.9 \\ \text { mean of |error } 1 \% \text { | } & 0.1 & 0.2 & 0.3 & 0.5\end{array}$


Figure 5 displays the approximation errors ("error\%") for the different correlation matrices $R_{i}, i=1,2,3$. As is proved in the theoretical part of the paper, "error" is zero at the general equilibrium $\bar{x}=\bar{y}=\bar{z}$. The other regions reveal errors not being much higher than in the previous case $\rho_{1}=\rho_{2}=\rho_{3}$. The largest deviations can be observed for $P \approx 1.1$ (which implies $\bar{X} / \bar{Y}=1.22$ ) in the case with heterogenous correlation coefficients $(R 3)$, where "error\%" is about $-7 \%$. 
Figure 5: Approximation errors ("error") under the assumption of general correlation structures

Homogeneous and smoll correlation coefficients (R2)

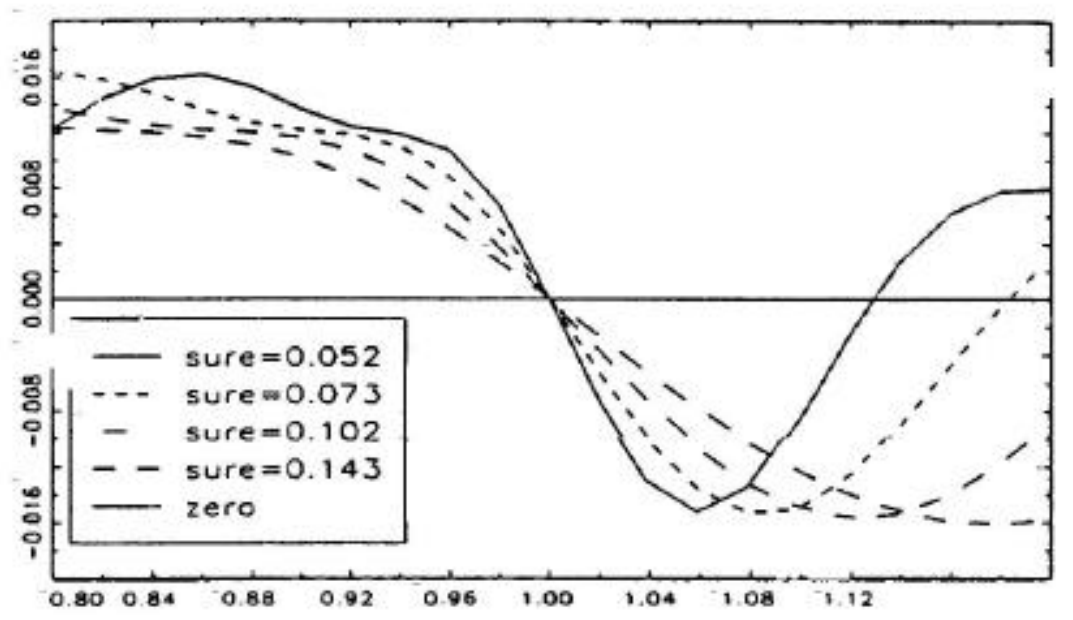

Homogeneous and large correlation coefficients (R2)
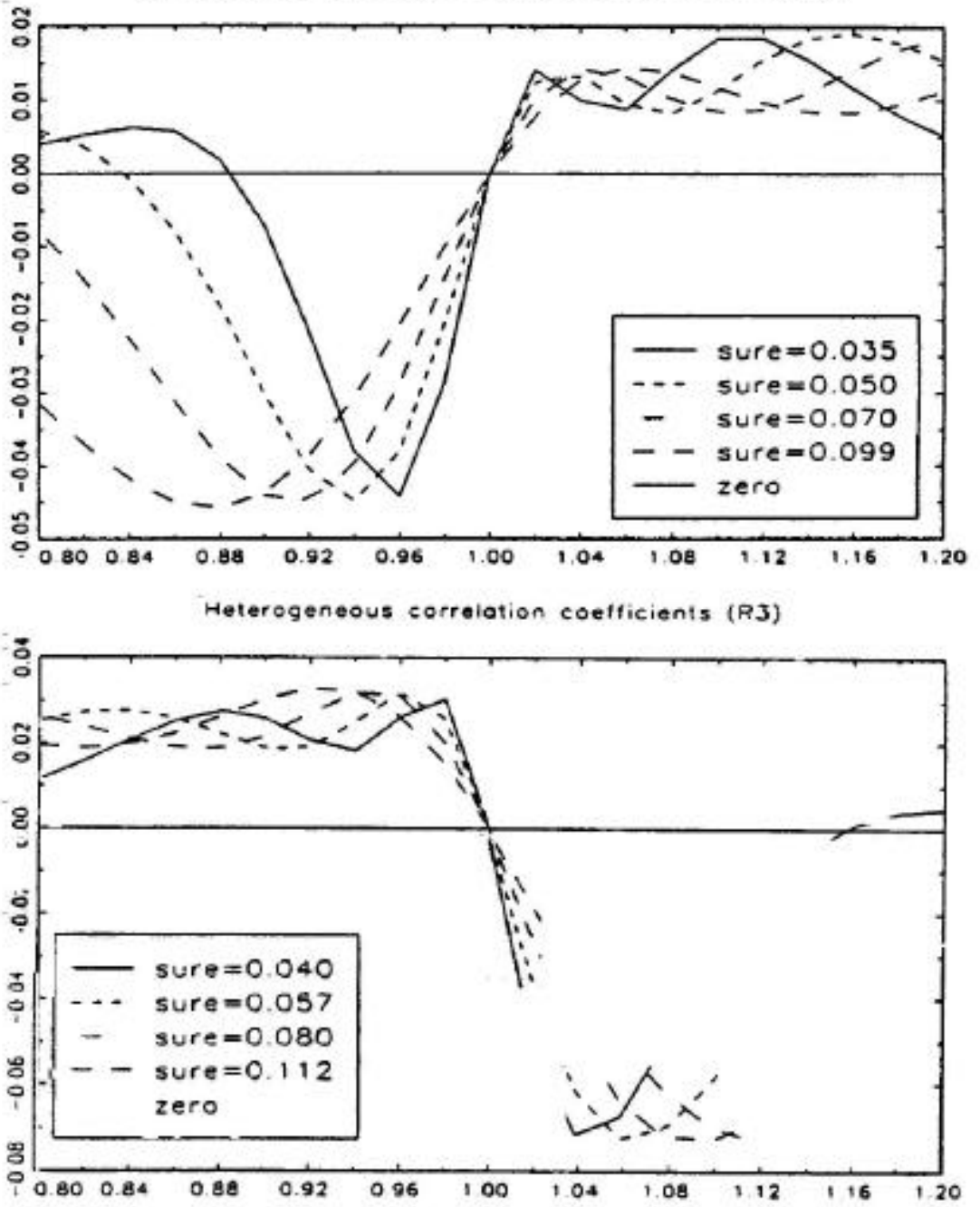

Note: See the text for the definition of underlying variance-covariance matrices 


\section{Concluding Remarks}

This paper is concerned about the estimation of aggregate employment functions in QRM models, when the diversity of situations across micromarkets is explicitly recognized. The aggregation result given in the paper generalizes that of Lambert (1988) to employment functions with more than two components, and leads to approximate aggregate functions of the CES variety. The approximation used can accomodate general variance-covariance structures. The simulation experiments have shown that the approximation error remains within reasonable bounds (1-4\%). It thus seems that the CES formulation can accomodate a large variety of situations, much less restrictive than those corresponding to the exact case with Weibull distributions.

\section{References}

Barro, R.J., Grossman, H.I., (1971), "A General Disequilibrium Model of Income and Employment", American Economic Review, 61, 82-93.

Bernanke, B., M.Gertler, (1989), "Agency Costs, Net Worth, and Business Cycle Fuctuations", American Economic Review, 79-1, 14-31.

Bierings, H., Muysken, J., (1988), "Nested Employment Functions and Structural Unemployment, with an Empirical Application to the Dutch and Belgian Economies", mimeo, Faculty of Economics and Business Administration, University of Limburg, Maastricht, The Netherlands.

Blanchard, O.J., S.Fischer, (1989), Lectures on Macroeconomics, MIT Press.

Carlstrom, C.T., T.S.Fuerst, (1997), "Agency Costs, Net Worth, and Business Cycle Fluctuations: A Computable General Equilibrium Analysis", American Economic Review, 87-5, 893-910.

Drèze, J.H., Bean, Ch., Lambert, J.P., Mehta, F., Sneessens, H.R., eds., (1990), Europe's Unemployment Problem, MIT Press.

Fagnart, J.F., O.Licandro, H.R.Sneessens, (1997), "Capacity Utilization and Market Power", Journal of Economic Dynamics and Control, 22,123-140.

Gouriéroux, C., Laffont, J.-J., Monfort, A., (1984), "Econométrie des modèles d'équilibre avec rationnement: une mise a jour", Annales de l'INSEE, 55/56, 5-38.

Heinesen, E., (1993), "CES Transaction Functions in Macroeconomic Rationing Models", mimeo, Institute of Economics, University of Copenhagen.

Heinesen, E., (1994), "CES Transaction Functions in Macroeconomic Rationing Models, Recherches Economiques de Louvain, 60(3), 301-331.

Heinesen, E., (1995), "The Two-Variable CES Transaction Function in Macroeconomic Rationing Models", Economics Letters, 48, 257-265.

Kahn, J., (1987), "Inventories and the Volatility of Production", American Economic Review, 77-4, 667-679.

Lambert, J.-P., (1988), Disequilibrium Macroeconomic Models, Cambridge University Press, Cambridge.

Malinvaud, E., (1977), The Theory of Unemployment Reconsidered, Basil Blackwell. 
Malinvaud, E., (1980), "Macroeconomic Rationing of Employment", in E. Malinvaud, J.P. Fitoussi, eds., Unemployment in Western Countries, Macmillan.

Muellbauer, J., (1978), "Macrotheory vs Macroeconometrics: The Treatment of Disequilibrium in Macro Models", Birkbeck DP 59, Birkbeck College, London.

Nilles, J., (1991), "Un modèle macroéconomique avec contraintes quantitatives et changement structurel", Master thesis, Department of Economics, Université Catholique de Louvain, Louvain-la-Neuve.

Smolny, W., (1993), Dynamic Factor Demand in a Disequilibrium Context. Theory and Estimation of a Macroeconomic Rationing Model for the Federal Republic of Germany, Springer-Verlag.

Sneessens, H.R., (1983), "Aggregation in Quantity Rationing Models", unpublished manuscript, Département des Sciences Economiques, Université Catholique de Louvain.

Sneessens, H.R., Drèze, J.H., (1986), "A Discussion of Belgian Unemployment, Combining Traditional Concepts and Disequilibrium Econometrics", Economica, 53, S89-S119. 


\section{Appendix}

$$
L=E[\min (x, y, z)]
$$

with

$$
\begin{aligned}
& x=e^{\bar{x}+u} \quad E(x)=\bar{X}=\exp \left\{\bar{x}+\frac{1}{2} \sigma_{u}^{2}\right\} \\
& y=e^{\bar{y}+v} \quad E(y)=\bar{Y}=\exp \left\{\bar{y}+\frac{1}{2} \sigma_{v}^{2}\right\} \\
& z=e^{\bar{z}+w} \quad E(z)=\bar{Z}=\exp \left\{\bar{z}+\frac{1}{2} \sigma_{w}^{2}\right\} \\
& (u, v, w) \sim N(0, \Sigma)
\end{aligned}
$$

We have $L=L_{1}+L_{2}+L_{2}$, where

$$
\begin{aligned}
& L_{1}=\int_{0}^{\infty} \int_{x}^{\infty} \int_{x}^{\infty} x f(x, y, z) d z d y d x \\
& L_{2}=\int_{0}^{\infty} \int_{y}^{\infty} \int_{y}^{\infty} y f(x, y, z) d z d x d y \\
& L_{3}=\int_{0}^{\infty} \int_{z}^{\infty} \int_{z}^{\infty} z f(x, y, z) d y d x d z
\end{aligned}
$$

Assume $\quad f(x, y, z)=\frac{(x y z)^{-1}}{2 \pi^{3 / 2}|\Sigma|^{1 / 2}} \exp \left\{-\frac{1}{2}(u v w) \Sigma^{-1}\left(\begin{array}{l}u \\ v \\ w\end{array}\right)\right\}$.

Proceed to the following change of variable:

$$
\left(\begin{array}{l}
\ln x \\
\ln y \\
\ln z
\end{array}\right)=\left(\begin{array}{l}
\bar{x} \\
\bar{y} \\
\bar{z}
\end{array}\right)+\left(\begin{array}{l}
u \\
v \\
w
\end{array}\right), \quad J=\left(\begin{array}{lll}
\frac{\partial u}{\partial x} & \frac{\partial u}{\partial y} & \frac{\partial u}{\partial z} \\
\frac{\partial v}{\partial x} & \frac{\partial v}{\partial y} & \frac{\partial v}{\partial z} \\
\frac{\partial w}{\partial x} & \frac{\partial w}{\partial y} & \frac{\partial w}{\partial z}
\end{array}\right)=\left(\begin{array}{ccc}
x^{-1} & 0 & 0 \\
0 & y^{-1} & 0 \\
0 & 0 & z^{-1}
\end{array}\right)
$$

Hence

$$
\begin{aligned}
& L_{1}=\int_{-\infty}^{\infty} \int_{\bar{x}-\bar{y}+u}^{\infty} \int_{\bar{x}-\bar{z}+u}^{\infty} e^{\bar{x}+u} g(u, v, w) d w d v d u \\
& L_{2}=\int_{-\infty}^{\infty} \int_{\bar{y}-\bar{x}+v}^{\infty} \int_{\bar{y}-\bar{z}+v}^{\infty} e^{\bar{y}+v} g(u, v, w) d w d u d v \\
& L_{3}=\int_{-\infty}^{\infty} \int_{\bar{z}-\bar{x}+w}^{\infty} \int_{\bar{z}-\bar{y}+w}^{\infty} e^{\bar{z}+w} g(u, v, w) d v d u d w
\end{aligned}
$$


where

$$
g(u, v, w)=\frac{1}{2 \pi^{3 / 2}|\Sigma|^{1 / 2}} \exp \left\{-\frac{1}{2}(u v w) \Sigma^{-1}\left(\begin{array}{l}
u \\
v \\
w
\end{array}\right)\right\}
$$

$L_{1}$ is then further transformed as follows (the same procedure will apply to $L_{2}$ and $L_{3}$ ):

$$
L_{1}=e^{\bar{x}} \int_{-\infty}^{\infty} \int_{\bar{x}-\bar{y}+u}^{\infty} \int_{\bar{x}-\bar{z}+u}^{\infty}\left[\frac{1}{2 \pi^{3 / 2}|\Sigma|^{1 / 2}} \exp \left\{-\frac{1}{2}\left(\varepsilon^{\prime} \Sigma^{-1} \varepsilon-2 u\right)\right\}\right] d w d v d u
$$

One can verify that $\varepsilon^{\prime} \Sigma^{-1} \varepsilon-2 u=(\varepsilon-\bar{\varepsilon})^{\prime} \Sigma^{-1}(\varepsilon-\bar{\varepsilon})-\sigma_{u}^{2}$ where $\bar{\varepsilon}=\Sigma\left(\begin{array}{l}1 \\ 0 \\ 0\end{array}\right)=\left(\begin{array}{l}\sigma_{u}^{2} \\ \sigma_{u v} \\ \sigma_{u w}\end{array}\right)$. Indeed

$$
\begin{aligned}
(\varepsilon-\bar{\varepsilon})^{\prime} \Sigma^{-1}(\varepsilon-\bar{\varepsilon}) & =\varepsilon^{\prime} \Sigma^{-1} \varepsilon-2 \varepsilon^{\prime} \Sigma^{-1} \bar{\varepsilon}+\bar{\varepsilon}^{\prime} \Sigma^{-1} \bar{\varepsilon} \\
& =\varepsilon^{\prime} \Sigma^{-1} \varepsilon-2 \varepsilon^{\prime} \Sigma^{-1} \Sigma\left(\begin{array}{l}
1 \\
0 \\
0
\end{array}\right)+\left(\begin{array}{lll}
1 & 0 & 0
\end{array}\right) \Sigma\left(\begin{array}{l}
1 \\
0 \\
0
\end{array}\right) \\
& =\varepsilon^{\prime} \Sigma^{-1} \varepsilon-2 u+\sigma_{u}^{2}
\end{aligned}
$$

Hence

$$
L_{1}=e^{\bar{x}+\sigma_{u}^{2} / 2} \int_{-\infty}^{\infty} \int_{\bar{x}-\bar{y}+u}^{\infty} \int_{\bar{x}-\bar{z}+u}^{\infty}\left[\frac{1}{2 \pi^{3 / 2}|\Sigma|^{1 / 2}} \exp \left\{-\frac{1}{2}(\varepsilon-\bar{\varepsilon})^{\prime} \Sigma^{-1}(\varepsilon-\bar{\varepsilon})\right\}\right] d w d v d u
$$

We again proceed to a change of variables: $\eta=\varepsilon-\bar{\varepsilon}=\left[\left(u-\sigma_{u}^{2}\right)\left(v-\sigma_{u v}\right)\left(w-\sigma_{u w}\right)\right]^{\prime}$

so that

$$
L_{1}=e^{\bar{x}+\sigma_{u}^{2} / 2} \int_{-\infty}^{\infty} \int_{\bar{x}-\bar{y}+\eta_{1}+\sigma_{u}^{2}-\sigma_{u v}}^{\infty} \int_{\bar{x}-\bar{z}+\eta_{1}+\sigma_{u}^{2}-\sigma_{u v}}^{\infty} h(\eta) d \eta_{3} d \eta_{2} d \eta_{1}
$$

where

$$
h(\eta)=\frac{1}{2 \pi^{3 / 2}|\Sigma|^{1 / 2}} \exp \left\{-\frac{1}{2} \eta^{\prime} \Sigma^{-1} \eta\right\}
$$

and hence

$$
\begin{aligned}
L_{1} & =\bar{X} \operatorname{Pr}\left\{\left(\eta_{2}>\bar{x}-\bar{y}+\eta_{1}+\sigma_{u}^{2}-\sigma_{u v}\right) \cap\left(\eta_{3}>\bar{x}-\bar{z}+\eta_{1}+\sigma_{u}^{2}-\sigma_{u w}\right)\right\} \\
& =\bar{X} \operatorname{Pr}\left\{\left(\eta_{1}-\eta_{2}<-\bar{x}+\bar{y}-\sigma_{u}^{2}+\sigma_{u v}\right) \cap\left(\eta_{1}-\eta_{3}<-\bar{x}+\bar{z}-\sigma_{u}^{2}+\sigma_{u w}\right)\right\}
\end{aligned}
$$

The following change of variable has to be used: 


$$
\eta^{*}=\left(\begin{array}{c}
\eta_{1} \\
\eta_{1}-\eta_{2} \\
\eta_{1}-\eta_{3}
\end{array}\right)=A \eta, \quad \eta^{*} \approx N\left(0, A \Sigma A^{\prime}\right)
$$

with

$$
A=\left(\begin{array}{rrr}
1 & 0 & 0 \\
1 & -1 & 0 \\
1 & 0 & -1
\end{array}\right), \quad A \Sigma A^{\prime}=\left(\begin{array}{ccc}
\sigma_{u}^{2} & \sigma_{u}^{2}-\sigma_{u v} & \sigma_{u}^{2}-\sigma_{u w} \\
* & \sigma_{u}^{2}+\sigma_{v}^{2}-2 \sigma_{u v} & \sigma_{u}^{2}-\sigma_{u v}-\sigma_{u w}+\sigma_{v w} \\
* & * & \sigma_{u}^{2}+\sigma_{w}^{2}-2 \sigma_{u w}
\end{array}\right)
$$

It follows that

$$
\begin{aligned}
L_{1} & =\bar{X} \int_{-\infty}^{\infty} \int_{-\infty}^{-\bar{x}+\bar{y}-\sigma_{u}^{2}+\sigma_{u v}} \int_{-\infty}^{-\bar{x}+\bar{z}-\sigma_{u}^{2}+\sigma_{u w}} h^{*}\left(\eta^{*}\right) d \eta_{3}^{*} d \eta_{2}^{*} d \eta_{1}^{*} \\
& =\bar{X} \int_{-\infty}^{-\bar{x}+\bar{y}-\sigma_{u}^{2}+\sigma_{u v}} \int_{-\infty}^{-\bar{x}+\bar{z}-\sigma_{u}^{2}+\sigma_{u v}} H^{*}\left(\eta_{2}^{*}, \eta_{3}^{*}\right) d \eta_{3}^{*} d \eta_{2}^{*}
\end{aligned}
$$

with

$$
H^{*}\left(\eta_{2}^{*}, \eta_{3}^{*}\right)=\int_{-\infty}^{\infty} h^{*}\left(\eta^{*}\right) d \eta_{1}^{*} .
$$

Next let us normalize:

$$
\begin{array}{ll}
\left(\begin{array}{l}
\eta_{2}^{*} \\
\eta_{3}^{*}
\end{array}\right) \approx N\left(0, V^{*}\right), & V^{*}=\left(\begin{array}{cc}
\sigma_{u}^{2}+\sigma_{v}^{2}-2 \sigma_{u v} & \sigma_{u}^{2}-\sigma_{u v}-\sigma_{u w}+\sigma_{v w} \\
* & \sigma_{u}^{2}+\sigma_{w}^{2}-2 \sigma_{u w}
\end{array}\right) \\
\left(\begin{array}{l}
\tilde{\eta}_{2} \\
\tilde{\eta}_{3}
\end{array}\right)=P^{-1 / 2}\left(\begin{array}{l}
\eta_{2}^{*} \\
\eta_{3}^{*}
\end{array}\right), & P=\left(\begin{array}{cc}
\sigma_{u}^{2}+\sigma_{v}^{2}-2 \sigma_{u v} & 0 \\
0 & \sigma_{u}^{2}+\sigma_{w}^{2}-2 \sigma_{u w}
\end{array}\right)=\left(\begin{array}{cc}
\sigma_{2}^{* 2} & 0 \\
0 & \sigma_{3}^{* 2}
\end{array}\right)
\end{array}
$$

Therefore, $\left(\begin{array}{l}\tilde{\eta}_{2} \\ \tilde{\eta}_{3}\end{array}\right) \sim N(0, \tilde{V}), \quad \tilde{V}=E\left(\tilde{\eta} \tilde{\eta}^{\prime}\right)=\left(\begin{array}{cc}1 & \tilde{\rho}_{1} \\ \tilde{\rho}_{1} & 1\end{array}\right)$

with

$$
\tilde{\rho}_{1}=\frac{\sigma_{u}^{2}-\sigma_{u v}-\sigma_{u w}+\sigma_{v w}}{\sqrt{\sigma_{u}^{2}+\sigma_{v}^{2}-2 \sigma_{u v}} \sqrt{\sigma_{u}^{2}+\sigma_{w}^{2}-2 \sigma_{u w}}}=\frac{1}{2} \frac{\sigma_{2}^{* 2}+\sigma_{3}^{* 2}-\sigma_{4}^{* 2}}{\sigma_{2}^{*} \sigma_{3}^{*}} .
$$




\section{Therefore}

$$
\begin{aligned}
L_{1} & =\bar{X} \int_{-\infty}^{\frac{-\bar{x}+\bar{y}-\sigma_{u}^{2}+\sigma_{u v}}{\sigma_{2}^{*}}} \int_{-\infty}^{\frac{-\bar{x}+\bar{z}-\sigma_{u}^{2}+\sigma_{u w}}{\sigma_{3}^{*}}} \tilde{H}\left(\tilde{\eta}_{2}, \tilde{\eta}_{3}\right) d \tilde{\eta}_{3} d \tilde{\eta}_{2}, \quad \tilde{H}\left(\tilde{\eta}_{2}, \tilde{\eta}_{3}\right)=\sigma_{2}^{*} \sigma_{3}^{*} H^{*}\left(\tilde{\eta}_{2}, \tilde{\eta}_{3}\right) \\
& =\bar{X} F\left(\frac{-\bar{x}+\bar{y}-\sigma_{u}^{2}+\sigma_{u v}}{\sigma_{2}^{*}}, \frac{-\bar{x}+\bar{z}-\sigma_{u}^{2}+\sigma_{u w}}{\sigma_{3}^{*}} \mid \tilde{\rho}_{1}\right) \\
& =\bar{X} F\left(\frac{-\left(\bar{x}+\sigma_{u}^{2} / 2\right)+\left(\bar{y}+\sigma_{v}^{2} / 2\right)-\left(\sigma_{u}^{2}+\sigma_{v}^{2}-2 \sigma_{u v}\right) / 2}{\sigma_{2}^{*}},\right. \\
& =\bar{X} F\left(x_{2}^{*}-\sigma_{2}^{*} / 2, x_{3}^{*}-\sigma_{3}^{*} / 2 \mid \tilde{\rho}_{1}\right) \\
\text { with } \left.\quad \sigma_{2}^{2} / 2\right)+\left(\bar{z}+\sigma_{w}^{2} / 2\right)-\left(\sigma_{u}^{2}+\sigma_{w}^{2}-2 \sigma_{u w}\right) / 2 & \left.\sigma_{3}^{*}\right) \\
x_{2}^{*} & =\frac{-\left(\bar{x}+\sigma_{u}^{2} / 2\right)+\left(\bar{y}+\sigma_{v}^{2} / 2\right)}{\sigma_{2}^{*}}, \quad x_{3}^{*}=\frac{-\left(\bar{x}+\sigma_{u}^{2} / 2\right)+\left(\bar{z}+\sigma_{w}^{2} / 2\right)}{\sigma_{3}^{*}} .
\end{aligned}
$$

Also, $\quad \sigma_{2}^{*} x_{2}^{*}=\ln \bar{Y}\left|\bar{X}, \quad \sigma_{3}^{*} x_{3}^{*}=\ln \bar{Z}\right| \bar{X}$.

Similarly one can obtain $\quad L_{2}=\bar{Y} F\left(-x_{2}^{*}-\sigma_{2}^{*} / 2, \quad y_{3}^{*}-\sigma_{4}^{*} / 2 \mid \tilde{\rho}_{2}\right)$

with

$$
\begin{aligned}
& \sigma_{4}^{* 2}=\sigma_{v}^{2}+\sigma_{w}^{2}-2 \sigma_{v w} \\
& \tilde{\rho}_{2}=\frac{\sigma_{v}^{2}-\sigma_{u v}-\sigma_{v w}+\sigma_{u w}}{\sigma_{2}^{*} \sigma_{4}^{*}}=\frac{1}{2} \frac{\sigma_{2}^{* 2}-\sigma_{3}^{* 2}+\sigma_{4}^{* 2}}{\sigma_{2}^{*} \sigma_{4}^{*}} \\
& y_{1}^{*}=\frac{\left(\bar{x}+\sigma_{u}^{2} / 2\right)-\left(\bar{y}+\sigma_{v}^{2} / 2\right)}{\sigma_{2}^{*}}, \quad y_{3}^{*}=\frac{-\left(\bar{y}+\sigma_{v}^{2} / 2\right)+\left(\bar{z}+\sigma_{w}^{2} / 2\right)}{\sigma_{4}^{*}}
\end{aligned}
$$

and

$$
L_{3}=\bar{Z} F\left(-x_{3}^{*}-\sigma_{3}^{*} / 2, \quad-y_{3}^{*}-\sigma_{4}^{*} / 2 \mid \tilde{\rho}_{3}\right)
$$

with

$$
\tilde{\rho}_{3}=\frac{1}{2} \frac{\sigma_{3}^{* 2}-\sigma_{2}^{* 2}+\sigma_{4}^{* 2}}{\sigma_{3}^{*} \sigma_{4}^{*}}
$$

Also,

$$
\sigma_{4}^{*} y_{3}^{*}=\ln \bar{Z} / \bar{Y}=\sigma_{3}^{*} x_{3}^{*}-\sigma_{2}^{*} x_{2}^{*} \text {. }
$$

Finally,

$$
L=E[\min (x, y, z)]=\bar{X} F_{1}+\bar{Y} F_{2}+\bar{Z} F_{3} .
$$

